\title{
Sixth Sense Jacket
}

\author{
Prabhat S. Pandey ${ }^{\mathrm{a}}$ \\ prabhatpandey@outlook.com
}

\author{
Jay S. Gaikwad ${ }^{\text {a }}$ \\ jaygaikwad121@gmail.com
}

\author{
Shreya S. Chakraborty ${ }^{\text {a }}$ \\ shreya.04chakraborty@gmail.com
}

\author{
Divya K. Shah ${ }^{\text {a }}$ \\ divya.shah@rait.ac.in
}

${ }^{a}$ Department of Electronics Engineering, Ramrao Adik Institute of Technology, Nerul, Navi Mumbai,400 706,India

\begin{abstract}
In a time when the entire world is going through a pandemic which can be curbed or substantially avoided and reduced by maintaining distance, it is crucial for every individual to follow the social distancing norms. However, it can be a challenging and daunting task to ensure safe distance all the time. Thus a Sixth Sense Jacket is a great way to keep the user mindful and alert of their surrounding without actually being alert all the time. It can not only alert the user but can also keep daily essentials such as keys and cards clean using dry sanitization. The jacket comes with a bunch of special features such as Pulse Oximeter which can be used to check vital health stats such as heart rate and blood saturation $\left(\mathrm{SpO}_{2}\right)$ levels. The prime purpose of the Sixth Sense Jacket is to keep the user passively mindful of it's surrounding all the time and thus can be used in any field where it is crucial to stay alert in a given surrounding. The various domains this jacket can be used are women safety, battlefield and to add an extra sense to visually impaired people. The jacket can be a useful means to pre-empt any given danger that might befall the user and hence the name "Sixth Sense" Jacket.
\end{abstract}

Index Terms-Social Distancing, PIR Sensor, Raspberry Pi Zero, Dry sanitization, Pulse Oximeter, MAX30100, Sixth Sense

\section{INTRODUCTION}

In the recent times, all have realized the importance of social distancing while in public or in crowded places. So a safety jacket would help the person wearing it maintain social distancing more effectively with the help of some electronics devices and components. The implementation of this jacket or the Sixth Sense Jacket will be based on the Raspberry Pi Zero single board computer which is a small, lightweight device and is highly compatible with our application. The jacket comes with additional features such as Ultraviolet LEDs in the outer pocket of the jacket. UV light is an effective method for dry sanitization and can be effectively used for sanitization of day to day object. Along with this a Pulse Oximeter Sensor which would deliver an output on a LED display is attached on the sleeve of the Jacket.

The main objective behind the designing of the sixth sense jacket is to allow people go out into the world because sometimes it is absolutely inevitable and one has to go out of the safety of their homes. In such a situation, this jacket will enable the user to stay mindful of his or her surrounding and at the same time keep a check of their health parameters by measuring and displaying various health vitals such as heart rate and Oxygen saturation levels. This way the user can be rest assured that he or she is taking all the necessary precautions amidst the pandemic.

\section{EXISTING TECHNIQUES}

Development Paper of a Sensing Module for Standing and Moving Human Body Use the Shutter and PIR Sensor to improve the sensor and body sensor module using Shutter and pyroelectric infrared (PIR) sensor ${ }^{[7]}$. Test results show that the hearing distance is 7.0 meters hearing angles around 110 degrees at room temperature. In these cases, the transmission rate is $100 \%$ and the power distribution of the module is rated as $100 \mathrm{~m}$.

Human Movement Detection and Identification Using Pyroelectric Infrared Sensors ${ }^{[8]}$. With three PIR-based modules in the monitoring hall; one roof module; two modules on opposite walls facing each other. Data were collected from eight subjects when traveling in three different, three intervals and three speed levels. Two types of feature sets were used: a raw data set and a reduced feature set made for size and height. The findings show that with the setting of raw data taken from one PIR sensor in three modules, more than $92 \%$ accuracy in distinguishing index. And in addition to $94 \%$ accuracy in classification, speed and distance identification using a reduced feature set extracted from two PIR sensors for each of the three modules.

Modifying UV blocking effect using polyester fabric ${ }^{[2]}$. The influence of conventional thermal and alkaline dyeing processes on fabric formation is being investigated. A spectrophotometer with a connecting sphere is used to measure the UV transfer of polyester fabrics according to AATCC tests. The slight difference in the weight of the fabrics after the blinding processes already affects the UV transfer of the fabrics. Orange and blue dyed fabrics show a high enough UPF, while pale red fabric does not achieve such values. Double-dyed fabrics with both fabrics after being treated with a UV absorber reach high UPF values.

Low Cost Pulse Oximetry Design Based on the Raspberry Pi. Pulse oximetry is a non-invasive method of monitoring oxygen saturation ${ }^{[1]}$. Oxygen intake $\left(\mathrm{SpO}_{2}\right)$ 
is a percentage of oxygen-containing hemoglobin. Pulse Oximetry operates on the condition that hemoglobin with dissolved oxygen $\left(\mathrm{HbO}_{2}\right)$ captures light at different wavelengths from hemoglobin that does not bind oxygen $(\mathrm{Hb})$. This method uses different wave- lengths of red light $(660 \mathrm{~nm})$ and infrared light $(940 \mathrm{~nm})$ carried by the light sensor after passing through the veins and bones at the end of the index finger ${ }^{[8]}$.

\section{BLOCK DIAGRAM}

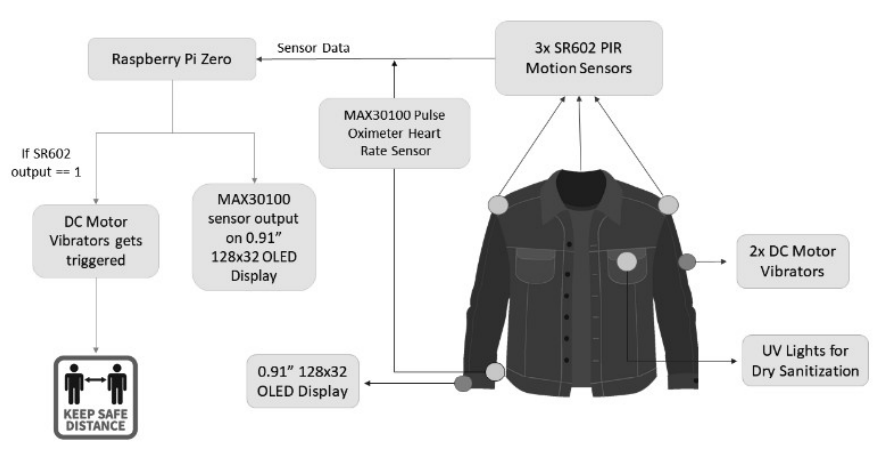

Fig. 1. Block Diagram

The Sixth Sense Jacket incorporates various features for achieving its purpose, depicted using the block diagram(fig.1.).

Distancing Mechanism: The Sixth Sense Jacket has three SR602 PIR Sensors. One sensor each on the left and the right shoulder of the jacket and the third one on the back, below the collar of the jacket. The output of the PIR sensor will be reflected on the DC Motor Vibrators. Four such DC motor vibrators are used in this project, two vibrators each on the right and left arm of the jacket.

Health Stats Mechanism: One MAX30100 Pulse Oximeter Heart Rate Sensor is being used along with an 0.91 " OLED display for displaying the data accumulated by the PO Sensor. The MAX30100 and OLED Display will be mounted on a wide wrist band, with the display on the upper end of band visible to the user.

Dry Sanitization: The UV Light which will be used for dry sanitization will be in the outer pocket of the jacket, it will be controlled manually using a switch. For safety purpose, the pockets will be coated with a thick layer of dyed polyester fabric to prevent the UV Light's effect on the user.

\section{WORKING}

By referring the circuit diagram (fig. 2), the circuitry of the project can be understood. The sensors are setup when the Raspberry $\mathrm{Pi}$ initializes. The SR602 gives conditional output. If the sensor gets a reading which is less than 5 meters then the output will be triggered and the DC motor will start vibrating. There are three PIR sensors to be mounted on the left shoulder, the right shoulder and the backside of the jacket

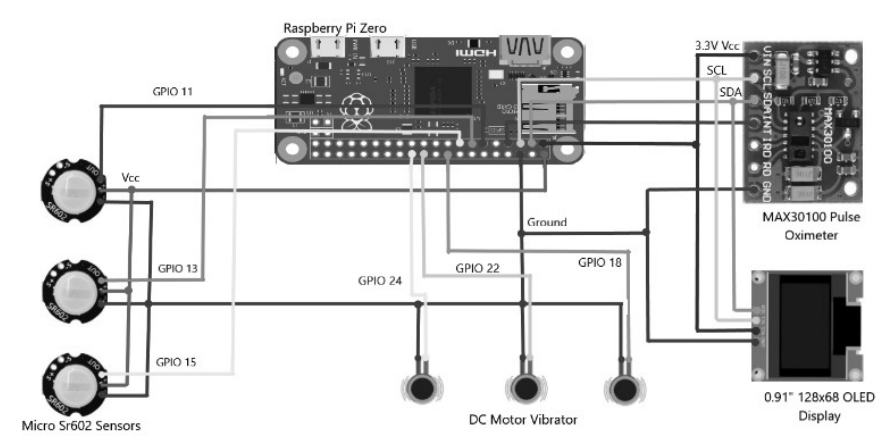

Fig. 2. Circuit Diagram

while there are two DC motor vibrators mounted on each arm of the jacket, all of them are connected on separate GPIO pins on the RPi. So if the sensor on the right side of the jacket gets an input within the range then the two DC motors on the right arm of the jacket will start vibrating thereby alerting the user, similarly with the left arm sensor triggers the motors on the left arm. In the situation when someone is approaching from the back, all the four DC motor vibrators will be triggered simultaneously alerting the user.

For the MAX30100 Pulse Oximeter sensor no GPIO pins needs to be allotted but the sensor needs to be connected to the SDA (Serial Data Line) and SCL (Serial Clock Line) of the Raspberry Pi along with the SDA and SCL of the OLED display. So as the PO sensor starts reading the pulse reading and the oxygen saturation of the user, the output will be received on the OLED display in real-time. The PO sensor will be fixed on the wrist of the user using a band, while the OLED Display will be mounted right on the opposite side of the band. For the purpose of power consumption a 2 pin switch can be allotted to turn this peripheral off. Proper flow of all the process of the project can be clearly understood by referring the flow-diagram (fig.3.).

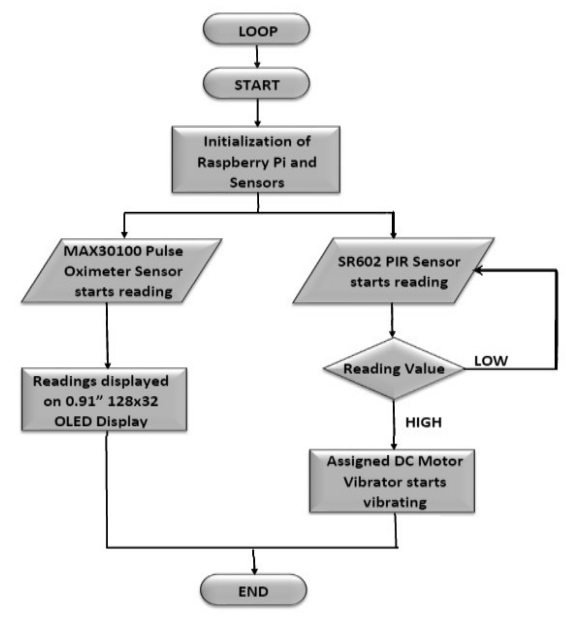

Fig. 3. Flow-diagram 


\section{FLOWCHART}

The flowchart (fig. 4) represents the working of the SR602 PIR Sensor. It shows the condition of the three sensors which will be on the left shoulder, right shoulder and the back of the user. All the sensors will run in a loop, meaning the PIR sensor will continuously fetch the data within the range of less than $200 \mathrm{~cm}$ in this case $(\mathrm{cm}<=200)$. Depending on the location where the jacket is to be used, the unit can be modified by very minor changes in the code.

The 'readCentimeter' parameter is being used here for detecting the distance, so as soon the condition becomes true, the DC Motor Vibrators assigned to each sensor gets triggered thereby alerting the user. The sensor on the left shoulder and the right shoulder will be assigned two DC motor vibrators each whereas for producing the output for the sensor on the back of the jacket, all the four DC motor vibrators are assigned. LOOP3 below represents the sensor on the backside of the jacket whereas LOOP1 and LOOP2 depicts the sensor on either of the left or right shoulder.

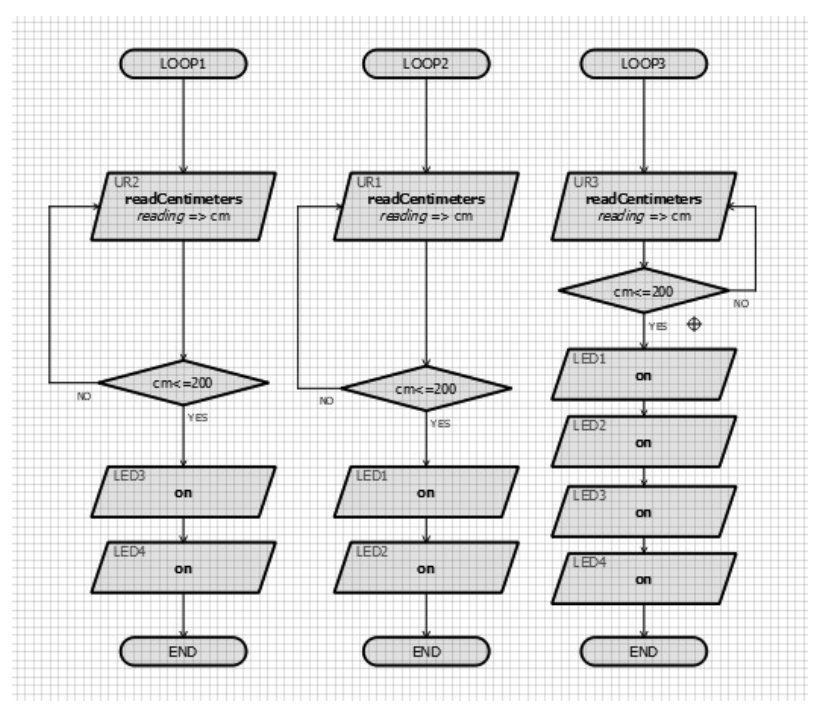

Fig. 4. Flowchart

\section{Results}

The integration of the components results in desired output i.e. the SR602 sensors giving accurate output if a human comes within the range $(<=5$ meters $)$, the range can be adjusted by some minor changes in the code or by mounting a potentiometer on the sensor itself that will adjust its sensitivity. The output of PIR sensor triggers the assigned DC Motor Vibrator which vibrates thereby alerting the user. The SR602 has considerably less power consumption of $20 \mathrm{uA}$ and also has an accuracy of over $94 \%{ }^{[8]}$. For getting a depth of power requirement of the jacket, the current and voltage ratings of all the devices can be referred from the datasheet (fig. 5.).

\begin{tabular}{|l|c|c|c|c|}
\hline \multicolumn{1}{|c|}{ Ratings } & Voltage & Current & Pieces & $\begin{array}{c}\text { Total } \\
\text { Consumption }\end{array}$ \\
\hline $\begin{array}{l}\text { Components } \\
\text { Sensor PIR Motion }\end{array}$ & $3.3 \mathrm{~V}$ & $0.02 \mathrm{~mA}$ & 3 & $0.06 \mathrm{~mA}$ \\
\hline $\begin{array}{l}\text { MAX30100 PO Heart } \\
\text { Rate Sensor }\end{array}$ & $5 \mathrm{~V}$ & $1.2 \mathrm{~mA}$ & 1 & $1.2 \mathrm{~mA}$ \\
\hline 0.91 " OLED Display & $5 \mathrm{~V}$ & $2 \mathrm{~mA}$ & 1 & $2 \mathrm{~mA}$ \\
\hline DC Motor Vibrators & $3 \mathrm{~V}$ & $80 \mathrm{~mA}$ & 4 & $320 \mathrm{~mA}$ \\
\hline Raspberry Pi Zero & $3.3 \mathrm{~V}$ & $120 \mathrm{~mA}$ & 1 & $120 \mathrm{~mA}$ \\
\hline Net Charge Consumption (Ideal state) & & & $=450 \mathrm{~mA}$ \\
\hline
\end{tabular}

Fig. 5. Datasheet

The MAX30100 Pulse Oximeter Sensor apart from the 5V Vcc and GND simply occupies the SDA and SCL pins. of the Raspberry $\mathrm{Pi}$ which is then shorted with the SDA and SCL terminal of the 0.91" OLED Display. This minimal connection makes the function of heart rate module easy and less complex additionally reducing the strain on Raspberry Pi.

\author{
Calculation: \\ Battery Capacity (mAh): 10000 \\ Net Charge Consumption (mA): 450 \\ Formula: $\quad$ Charge/discharge time (in Hours) $=\frac{\text { Amp-hour rating }}{\text { Continuous current (in Amps) }}$ \\ Here, Amp-hour rating of the battery $=10000 \mathrm{mAh}$ \\ Continuous current $($ charge consumption $)=450 \mathrm{~mA}$ \\ Hence, Discharge time $=22$ hours 13 mins
}

Fig. 6. Calculation

After analysis and calculation (fig. 6) it can be noticed that an $10,000 \mathrm{mAh}$ power bank is capable enough to fulfil the power requirements of the Sixth Sense Jacket for 22+ hours in ideal conditions, considering that all the sensors and the output device will be functioning constantly. The UV Light is not taken into consideration as ideally it may be used for a very short span. The charge consumption can be further lowered by using high end sensors and output devices which may increase the expense of the jacket.

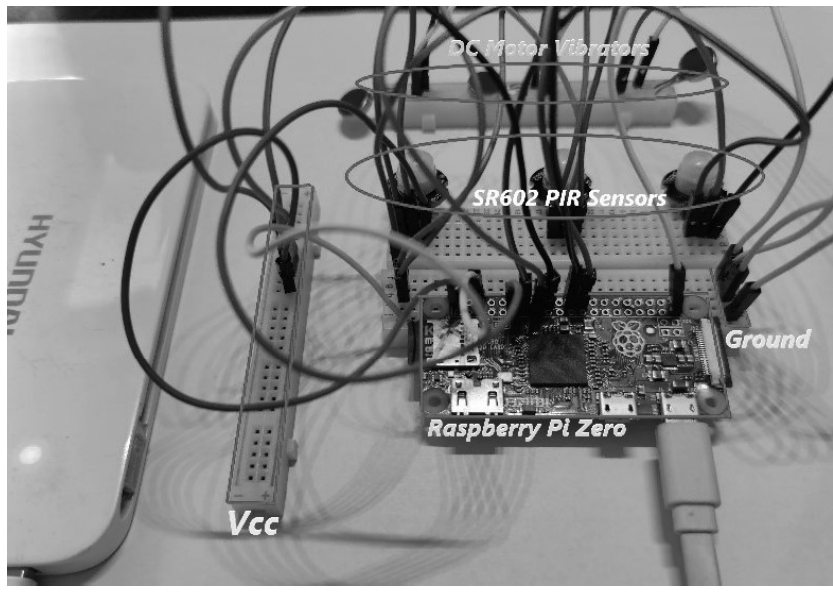

Fig.7. Hardware Assembly 
Proteus Design Suite is being used for the simulation (fig. 8.) of the SR602. As the library of the PIR sensor is unavailable for Raspberry Pi in Proteus, Grove Ultrasonic sensors are used for demonstrating the distancing mechanism of the jacket. When one of the sensor, representing the SR602 of the left or shoulder gets an reading of distance less than $200 \mathrm{~cm}$, the 2 LED lights glow up which represents the two assigned DC motor vibrators.

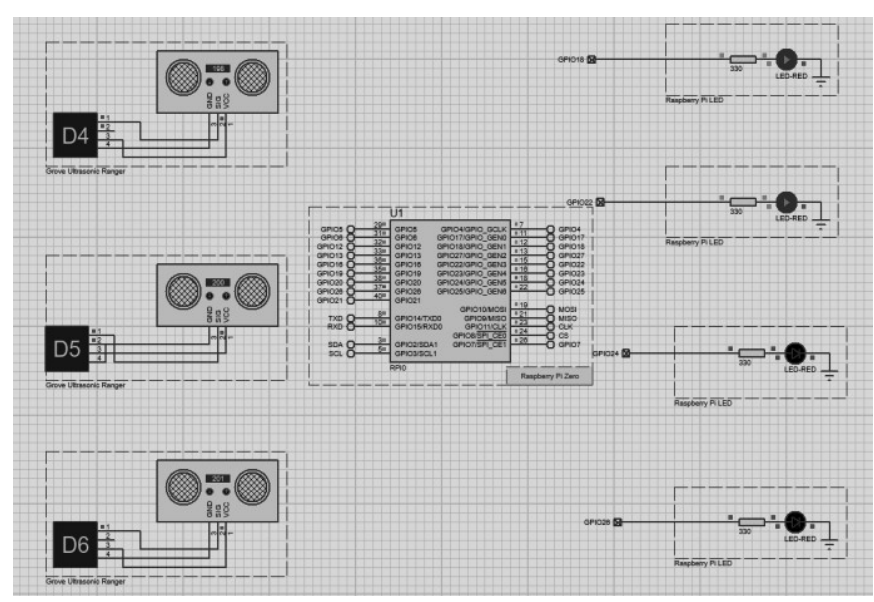

Fig. 8. Simulated Results

\section{VII.CONCLUSION}

The Sixth Sense Jacket is everything one can need in today's situation. The concept of the jacket is not very complex which makes the jacket very basic yet user friendly and affective when it comes to daily use. The sensors SR602 and MAX30100 are really cost effective and compact. In total there are $8 \mathrm{I} / \mathrm{O}$ devices yet the charge consumption of the devices are low enough to comfortably make if through the day. While this jacket may have few manageable drawbacks, the purpose and implementation can make this project notable. The biggest feature of the Sixth Sense Jacket is that it's not limited to a specific domain or task and the utility of the jacket can be ever-increasing.

\section{FUTURE SCOPE}

The Sixth Sense Jacket can have immense scope in present and future as well. The range of this jacket is vivid.

The number of PIR sensors can be increased enhancing the sensitivity of the jacket. On top of that a dedicated alerting mechanism can be created within a group of people wearing the Sixth Sense Jacket in a small geographical scale. An additional SOS feature can prove very useful for the military application of this jacket.

An IFTT based approach for alerting the user through phone alerts may prove beneficial by significantly reducing the number of output devices on the jacket thereby making space for more sensors that can be implemented on the same discharge time margin. The jacket if integrated with microphone and a compact speaker can be used for speak-to-translate feature in real-time. This feature will be an heavily IoT based approach and the audio device can simply be the audio device that will be in user by the user during the entire process.

Additionally it can be used for visually impaired people, women safety, construction sites and may more places where one needs to be alert.

\section{IX.REFERENCES}

1. S. Bakhri, E. Rosiana and RC Saputra. (2020). "Design of low cost pulse oximetry based on Raspberry Pi”. J. Phys.: Conf.Ser.1501012003.

2. Marija Gorensek and Franci Sluga. (June, 2014) "Modifying the UV blocking effects of polyester fabrics". Textile Research Journal, https://doi.org/10.1177/004051750407400601.

3. Yun J, and Sang-Shin Lee. 2014. "Human Movement Detection and Identification Using Pyroelectric Infrared Sensors" Sensors 14, no. 5: 8057-8081. https://doi.org/10.3390/s140508057

4. P. Zappi, E. Farella and L. Benini, "Tracking Motion Direction and Distance With Pyroelectric IR Sensors," in IEEE Sensors Journal, vol. 10, no. 9, pp. 1486-1494, Sept. 2010, doi: 10.1109/JSEN.2009.2039792.

5. Jian-Shuen Fang, Qi Hao, David J. Brady, Mohan Shankar, Bob D. Guenther, Nikos P. Pitsianis, and Ken Y. Hsu, "Path-dependent human identification using a pyroelectric infrared sensor and Fresnel lens arrays," Opt. Express 14, 609-624

(2006)

6. Mohan Shankar, John B. Burchett, Qi Hao, Bob D. Guenther, and David J. Brady "Human-tracking systems using pyroelectric infrared detectors," Optical Engineering 45(10), $106401 \quad(1 \quad$ October 2006). https://doi.org/10.1117/1.2360948

7. Ronnie O. Serfa Juan, Jin Su Kim, Yui Hwan Sa, Hi Seok Kim and Hyeong Woo Cha. "Development of a sensor module for standing and moving human body using a shutter and PIR Sensor".International Journal of Multimedia and Ubiquitous Engineering Vol.11,No.7(2016),pp.47-56. http://dx.doi.org/10.14257/ijmue.2016.11.7.05

8. E. Susana and H. Tjahjadi, "Handheld pulse oximeter based on single board computer raspberry Pi B +" $201715^{\text {th }}$ International Conference on Quality in Research (QiR) : International Symposium on Electrical and Computer Engineering, 2017, doi:10.1109/QIR.2017.8168470. pp.141-145, 